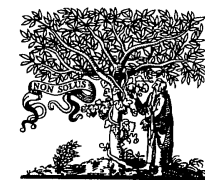

ELSEVIER
International Journal of Risk \& Safety in Medicine 7 (1995) $177-178$

\title{
Editorial
}

\section{The role of efficacy and risk in analgesia}

In general pharmacotherapy, efficacy is normally the prime consideration. Analgesics, curiously, seem to be an exception. Here incomplete or insufficient analgesia is tolerated even over long periods of time (notably in malignancies) because of the fear of inducing addiction if higher doses are employed. This holds true for all opiates and opioids, including propoxyphene, codeine and their derivatives. In less severe pain, the so-called "peripheral analgesics" are preferred for this reason, the term perhaps obscuring the fact that they exert a central effect too under certain conditions. The peripheral analgesic with the longest history of use in medicine, acetylsalicylic acid, definitely has no abuse potential and it has a clean record as regards nephrotoxicity. Even the many rheumatic patients who have taken an amount of this substance equalling their body weight have shown no renal impairment. There is, however, the problem of dose-dependent ulcerogenicity and, as we learned more recently, there is inhibition of thrombocyte aggregation which may increase the risk of dental and other surgery. Phenacetin with its proven nephrotoxicity (possible co-factors in that effect still being a matter for debate) had clear euphoriant properties. This is not true for the plethora of "antirheumatic" drugs of the acid type, but all these have more or less erosive effects on the gastro-intestinal mucosa, as well as affecting the regulation of the renal blood flow. Paracetamol alone has a relatively clean slate except when taken in excessive doses or by patients with already impaired liver function. With all "peripheral" analgesics it is indeed (except in individuals with hypersensitivity of allergic or idiosyncratic type) overdosage which constitutes the predominant risk. "Centrally acting" analgesics on the other hand tend to be either poorly tolerated (nausea, dysphoria) and are discontinued for that reason or else they lead to dependence; the latter, it should be added, is not regarded as a serious problem in patients with severe non-malignant pain or in cancer patients in whom the increasing dose required is solely of financial importance. One would therefore agree with the paper by Bannwarth et al. [2] that "peripherally acting" analgesics (NSAIDs) are the preparations of choice for light to moderate pain of limited duration (1-2 weeks) provided the patient has been prescribed only the recommended daily dose and has been asked to call immediately on his doctor if this dose does not work. The latter safeguard is naturally absent with the "over-the-counter" NSAIDs sold for self-medication. A recent report on segmental small-intestinal lesions in a patient with severe lumbosacral pain, who took up to 20 times $500 \mathrm{mg}$ mefenamic acid a day may illustrate this [1]. All patients with severe pain, the duration of which is as yet unknown, deserve to have this condition treated 
specifically and efficiently. If a chronic inflammatory process has to be treated at the same time, the situation will look different; but in degenerative arthrotic disease this is not the case.

Karl H. Kimbel Deputy Editor, Pharmaceuticals Hamburg, Germany

[1] Bielecki JW, Gebbers JO, Filippini L. Segmental small-intestinal lesions after taking NSAID's. Dtsch Med Wschr 1995;120:436-441.

[2] Bannwarth B, et al. NSAID's used in low doses as analgesics. Int J Risk Safety Med 1996; to be published. 